\title{
BOUNDING THE SIZE AND PROBABILITY OF EPIDEMICS ON NETWORKS
}

\author{
JOEL C. MILLER, ${ }^{*}$ British Columbia Centre for Disease Control
}

\begin{abstract}
We consider an infectious disease spreading along the edges of a network which may have significant clustering. The individuals in the population have heterogeneous infectiousness and/or susceptibility. We define the out-transmissibility of a node to be the marginal probability that it would infect a randomly chosen neighbor given its infectiousness and the distribution of susceptibility. For a given distribution of outtransmissibility, we find the conditions which give the upper (or lower) bounds on the size and probability of an epidemic, under weak assumptions on the transmission properties, but very general assumptions on the network. We find similar bounds for a given distribution of in-transmissibility (the marginal probability of being infected by a neighbor). We also find conditions giving global upper bounds on the size and probability. The distributions leading to these bounds are network independent. In the special case of networks with high girth (locally tree-like), we are able to prove stronger results. In general, the probability and size of epidemics are maximal when the population is homogeneous and minimal when the variance of in- or out-transmissibility is maximal.
\end{abstract}

Keywords: Epidemiology; network; attack rate; probability; transmissibility

2000 Mathematics Subject Classification: Primary 92D30

Secondary $60 \mathrm{~K} 35$

\section{Introduction}

The spread of infectious disease is governed by many different factors which vary on the individual level. Heterogeneity in the population comes from a number of sources including, but not limited to, genetic diversity, previous infections, vaccination history, or the existence of co-infections. In this paper we investigate the effects of heterogeneity on disease spread, focusing on the effect of simultaneous heterogeneities in infectiousness and susceptibility.

We consider the spread of infectious diseases in networks as shown in Figure 1. Individuals in the population are modeled as nodes and potentially infectious contacts are modeled as edges between the corresponding nodes. We consider the spread of an SIR disease, that is, the nodes are divided into three compartments: susceptible, infected, and recovered. A susceptible node may be infected by an infected neighbor. Following infection, the newly infected node may infect some, all, or none of its neighbors and then recover. After recovery, a node cannot be reinfected. Typically, in a large network outbreaks are either small or large (in a sense made more formal in Section 2). We are primarily interested in what controls the probability of large outbreaks and the fraction of nodes infected in a large outbreak.

Received 9 October 2007; revision received 15 April 2008.

* Postal address: $655 \mathrm{~W}$ 12th Avenue, Vancouver, BC V5Z 4R4, Canada.

Email address: joel.miller.research@gmail.com

Much of this work was completed while at the Center for Nonlinear Studies and the Mathematical Modeling \& Analysis Group, Los Alamos National Laboratory. 

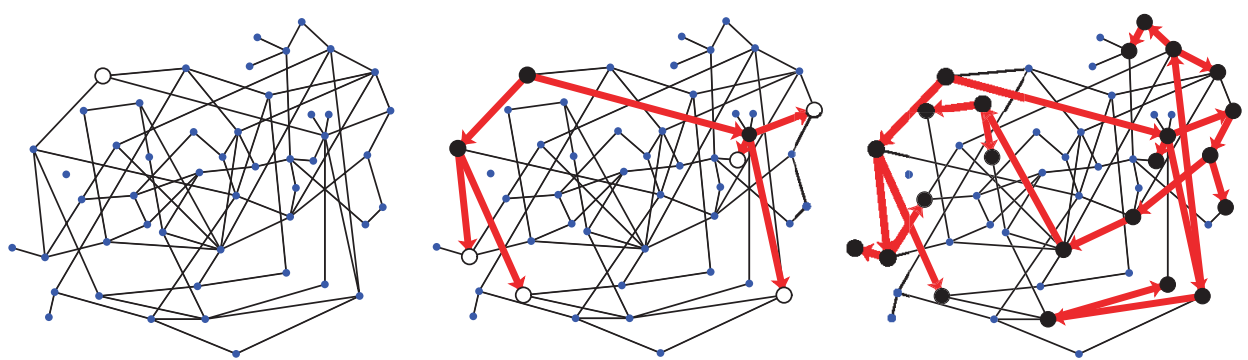

Figure 1: The spread of disease in a network. The outbreak begins with a single infected individual (large open circles) and then spreads along edges to others. The infected nodes recover with immunity (large filled circles). Eventually the outbreak dies out.

Before discussing earlier results, we introduce some terminology. The transmissibility $T_{u v}$ is the probability that an infection of node $u$ would result in the direct infection of the neighbor $v$. The in-transmissibility $T_{\mathrm{in}}(v)$ is the marginal probability that a neighbor of $v$ would infect $v$ given the characteristics of $v$, and the out-transmissibility $T_{\text {out }}(u)$ is the marginal probability that $u$ would infect a neighbor given the characteristics of $u$. Both the in- and outtransmissibility necessarily have the same average, $\langle T\rangle$. These definitions will be made more precise in Section 2.

Most network-based epidemic models assume homogeneous transmissibility $T_{u v}=\langle T\rangle$ between all pairs of neighboring nodes. Models that do allow heterogeneities generally show that they reduce the probability or size of epidemics [4], [13], [16], [21], [29]. For an arbitrary network with homogeneous susceptibility $\left(T_{\text {in }}(v)=\langle T\rangle\right.$ for all $\left.v\right)$, but heterogeneous infectiousness, Kuulasmaa [16] showed that epidemics are most likely and largest if infectiousness is homogeneous $\left(T_{\text {out }}(u)=\langle T\rangle\right.$ for all $u$ ). It was noted in [29] that the same argument shows that, with homogeneous susceptibility, epidemics are least likely and smallest if infectiousness is maximally heterogeneous ( $T_{\text {out }}=0$ for a fraction $1-\langle T\rangle$ of the population and $T_{\text {out }}=1$ for the remainder). The recent work of Kenah and Robins [13] and Miller [21] considered the effect of heterogeneity on a specific subclass of unclustered networks (variously called Molloy-Reed networks [23] or configuration model networks [26]), finding similar results. One of these, Miller [21], studied simultaneous heterogeneities in susceptibility and infectiousness, showing that, for given $\langle T\rangle$, the same cases give the upper and lower bounds on probability while epidemics are largest if susceptibility is homogeneous and smallest if susceptibility is maximally heterogeneous. We are unaware of any work which has considered simultaneous heterogeneities in infectiousness and susceptibility in networks with clustering or even in unclustered networks more general than Molloy-Reed networks.

In this paper we investigate the spread of epidemics in which $T_{\text {in }}$ and $T_{\text {out }}$ can simultaneously be heterogeneous, using techniques from [16] and [21]. We will consider both clustered and general unclustered networks. Clustered networks are more difficult because of the existence of short cycles, and so a stronger assumption will be made for them.

Often only the distribution of $T_{\text {out }}$ (or, more rarely, of $T_{\text {in }}$ ) would be available early in an outbreak. If we know the distribution of $T_{\text {out }}$, it does not in general uniquely determine the distribution of $T_{\text {in }}$ and so we cannot fully predict the final details of an outbreak. Our focus is on identifying the best and worst case scenarios given the distribution of $T_{\text {out }}$ (or $T_{\text {in }}$ ), thus helping to provide policy makers with knowledge of what to expect and how best to mitigate it. 
Mathematical theories modeling the spread of infectious diseases have been developed in a number of fields [1], [2], [8], [14]. The techniques used include differential equations, stochastic models, agent-based simulations, and network-based approaches. The differential equations approaches may be thought of as a mean-field approximation to a subclass of network models, while the stochastic and agent-based approaches can be made formally equivalent to networkbased methods [13], [24]. Consequently, results for networks will apply to other models as well. Network epidemic models have primarily been studied by the statistics community [3], [4], [5], [16], [17], [22], [30], and the statistical physics/applied mathematics communities [9], [10], [11], [18], [19], [20], [25], [27], [28]. In general, the statistics community has produced more rigorous results, but has considered more restricted classes of networks. The physics and applied mathematics communities have considered a wider range of networks, but the results are less rigorous. The interaction between these fields has been relatively sparse, leading to repeated discoveries of some results and a lack of cohesion in the topics studied. We attempt to bring some of these different approaches together in this paper.

This paper is structured as follows. In Section 2 we introduce the model and clarify definitions. In Section 3 we consider epidemics spreading on general networks. In Section 4 we find stronger results for networks with no short cycles. Finally, in Section 5 we discuss extensions and implications of our results.

\section{Epidemics in networks}

We consider the spread of disease on a network $G$. An outbreak begins when a single node (the index case) chosen uniformly from the population is infected. The disease spreads from an infected node $u$ to a neighboring susceptible node $v$ with a probability equal to the transmissibility $T_{u v}$. Each infected node attempts to infect each of its neighbors and then recovers (and is no longer susceptible or infected). The outbreak ends when no infected nodes remain.

This section is divided into three parts. First we describe the neighbor-to-neighbor transmissibility $T_{u v}$. This will depend on the characteristics of both $u$ and $v$. We then introduce the concept of an epidemic percolation network, which is a tool to study the routes of transmission in a given network. We finally discuss tools which will be used to make the concept of a 'large network' rigorous.

\subsection{Transmissibility}

Following [21], we assume that the factors influencing infectiousness of node $u$ and susceptibility of node $v$ may be summarized in $\mathcal{I}_{u}$ and $\delta_{v}$. In general, these may be vectorvalued functions (though with few exceptions they are taken to be scalars in the literature). For example, $\tau_{u}$ may represent $u$ 's viral load, duration of infection, and willingness or ability to leave work if sick, while $\delta_{v}$ may represent $v$ 's previous vaccination history, genetic predisposition to infection, and previous exposure to related infections. If $u$ and $v$ are neighbors, the transmissibility $T_{u v}$ is then

$$
T_{u v}=T\left(\mathcal{I}_{u}, \diamond_{v}\right)
$$

for some function $T$. The function $T(\mathcal{L}, \S)$ is the probability of transmission from a node with infectiousness $\mathcal{I}$ to a node with susceptibility $\delta$, assuming that the nodes are joined by an edge. We may think of $T_{u v}$ as defined only for neighboring nodes, or we may take $T_{u v}=\chi_{\{u, v\}} T\left(\mathcal{I}_{u}, \wp_{v}\right)$, where $\chi_{\{u, v\}}=0$ if $\{u, v\}$ is not an edge and $\chi_{\{u, v\}}=1$ if $\{u, v\}$ is an edge. 
We assume that $\mathcal{I}$ and $\delta$ are assigned independently, using the probability density functions $P(\mathcal{I})$ and $P(\&)$ (although we use the same symbol $P$ for both, we assume that the two functions are different). Particularly if $\mathcal{I}$ and $\delta$ are vectors, we may not be able to clearly define which of two nodes is 'more infectious' (i.e. there may not be a well-defined ordering). For example, with a sexually transmitted disease, we might have $u_{1}$ and $u_{2}$ infected, with $u_{1}$ having a high viral load and regular condom use (with occasional lapses), while $u_{2}$ has a low viral load but no condom use. Let us assume they have contacts with susceptibles $v_{1}$ and $v_{2}$, where $v_{1}$ has a high level of resistance, and, thus, will only be infected by a large dose, while $v_{2}$ has no immune protection and, thus, will be infected by even a small dose. Under these assumptions, $u_{1}$ is more likely to infect $v_{1}$, while $u_{2}$ is more likely to infect $v_{2}$. Which is 'more infectious' depends on the test susceptible considered.

The probability that $u$ infects a neighbor (prior to knowing $\&$ for that neighbor) is given by the out-transmissibility of $u$,

$$
T_{\text {out }}(u)=\int T\left(\mathcal{I}_{u}, \S\right) P(\S) \mathrm{d} \delta,
$$

and the probability that $v$ would be infected by a neighbor is given by the in-transmissibility of $v$,

$$
T_{\text {in }}(v)=\int T\left(\mathcal{L}, \wp_{v}\right) P(\mathcal{I}) \mathrm{d} \mathcal{I}
$$

At times it will be convenient to use $T_{\text {out }}(\mathcal{I})$ and $T_{\text {in }}(\mathcal{S})$ (rather than $T_{\text {out }}(u)$ and $T_{\text {in }}(v)$ ) to denote the out- and in-transmissibility of arbitrary nodes with $\mathcal{I}$ and $\&$, respectively. When the concepts of being 'more infectious' and 'more susceptible' are clearly defined, $T_{\text {in }}(\&)$ and $T_{\text {out }}(\mathcal{I})$ are invertible functions. However, because the ordering is not well defined in general, they may not be invertible. If they are invertible, it is often convenient to change variables and set $\mathcal{I}_{u}=T_{\text {out }}(u)$ or $\delta_{v}=T_{\text {in }}(v)$. We will do this frequently in Section 3, where we restrict our attention to cases where the ordering described above is well defined.

From $P(\mathscr{S})$ and $P(\mathcal{I})$, we may find the distributions of $T_{\text {in }}$ and $T_{\text {out }}$. We use $Q_{\text {in }}\left(T_{\text {in }}\right)$ to denote the probability density function for the in-transmissibility $T_{\text {in }}$ and $Q_{\text {out }}\left(T_{\text {out }}\right)$ to denote the probability density function for the out-transmissibility $T_{\text {out }}$. The averages $\int_{0}^{1} T_{\text {in }} Q_{\text {in }}\left(T_{\text {in }}\right) \mathrm{d} T_{\text {in }}$ and $\int_{0}^{1} T_{\text {out }} Q_{\text {out }}\left(T_{\text {out }}\right) \mathrm{d} T_{\text {out }}$ are both equal to $\langle T\rangle$.

Given distributions of $\mathcal{I}$ and $\delta$ and the function $T$, there is always a $Q_{\text {in }}$ and $Q_{\text {out }}$ pair that result. Also, given a $Q_{\text {in }}$ or a $Q_{\text {out }}$, it is always possible to find $P(\mathcal{L}), P(\mathscr{S})$, and $T$ that are consistent. For example, given any $Q_{\text {in }}$, for each node $v$, we assign a $T_{\text {in }}(v)$ from $Q_{\text {in }}$ and set $\delta_{v}=T_{\text {in }}(v)$. Then $T(\mathcal{I}, \&)=\delta$ is consistent with $Q_{\text {in }}$ and yields $Q_{\text {out }}\left(T_{\text {out }}\right)=\delta\left(T_{\text {out }}-\langle T\rangle\right)$, where $\delta(\cdot)$ is the Dirac delta function. This means that, for any distribution of in-transmissibility, it is possible that the infectiousness of nodes is homogeneous. Although it is possible to find a $Q_{\text {in }}$ for any $Q_{\text {out }}$ (and vice versa), not all $Q_{\text {out }}$ and $Q_{\text {in }}$ pairs are compatible. For example, if $Q_{\text {in }}=(1-\langle T\rangle) \delta\left(T_{\text {in }}\right)+\langle T\rangle \delta\left(T_{\text {in }}-1\right)$ (i.e. susceptibility is maximally heterogeneous) then the out-transmissibility must be homogeneously distributed; no other distribution is possible. This particular example will be important in Section 4.

Although in principle $\mathcal{I}$ and $\delta$ may be vector-valued, they frequently are assumed to be scalars with the transmissibility between two neighbors given by (see, for example, [7] and [21])

$$
T_{u v}=T\left(\mathcal{I}_{u}, \wp_{v}\right)=1-\exp \left(-\alpha \mathcal{I}_{u} \diamond_{v}\right)
$$

A number of disease models yield this form. For example: let $\alpha$ be the rate at which a virus from an infected person reaches a susceptible person. Let $\tau_{u}$ be the infectious period of $u$. Let 
$\S_{v}$ be the probability that a virus reaching $v$ causes infection. Then the probability $p$ that $v$ has not become infected satisfies $\dot{p}=-\alpha \S_{v} p$. Integrating this over the infectious period $\tau_{u}$ of $u$ yields (1).

We need one final concept related to the transmissibility. Let a node $u$ be given, and let $V=\left\{v_{1}, \ldots, v_{m}\right\}$ be a subset of the neighbors of $u$. Assume that we know $\vec{s}=\left(\varsigma_{v_{1}}, \ldots, \varsigma_{v_{m}}\right)$, but not $\tau_{u}$. Define

$$
\phi_{\text {in }}(V, \vec{\jmath})=\int \prod_{v \in V}\left(1-T\left(\mathcal{L}, \wp_{v}\right)\right) P(\mathcal{L}) \mathrm{d} \mathcal{L} .
$$

This is the probability that $u$ will not infect any node in $V$ given knowledge of $s$ for each $v \in V$, but marginalized over the possible values of $\mathcal{L}$ for $u$. We may similarly define

$$
\begin{aligned}
\psi_{\text {in }}(V) & =\int \phi_{\text {in }}(V, \vec{\jmath}) P(\vec{\jmath}) \mathrm{d} \vec{\jmath} \\
& =\int\left(1-T_{\text {out }}\right)^{|V|} Q_{\text {out }}\left(T_{\text {out }}\right) \mathrm{d} T_{\text {out }} .
\end{aligned}
$$

This is the probability that $u$ will not infect any $v \in V$ marginalized over $s$ of $v \in V$ and the values of $\mathcal{I}$ for $u$. If $|V|=1$ then $\psi_{\text {in }}(V)=1-\langle T\rangle$, which will be important later when we consider unclustered networks.

\subsection{Epidemic percolation networks}

Given a network $G$, the distributions $P(\mathcal{I})$ and $P(\mathcal{S})$, and the function $T(\mathcal{I}, \mathcal{S})$, we assign $I$ and $\delta$ to each node of $G$. We then create a new directed network $\&$ which is an epidemic percolation network (EPN) [12] as follows: the nodes of $\&$ are the nodes of $G$. For each edge $\{u, v\}$ of $G$, we place directed edges $(u, v)$ and $(v, u)$ into $\&$ with probability $T_{u v}$ and $T_{v u}$, respectively. The original network $G$ gives the paths a disease could follow, while a realization of $\&$ gives the paths the disease will follow (if given the chance) for a simulation.

The out-component of a given node $u$ found by assigning $\mathcal{I}$ and $\delta$ and generating an EPN comes from the same distribution as the nodes infected by the dynamic epidemic process described earlier with $u$ as the index case. The processes are formally equivalent.

To motivate some definitions, we assume that transmissibility is sufficiently high that there are nodes in $\mathcal{E}$ with giant in- or out-components [6]. We define $H_{\text {out }}$ to be those nodes with a giant in-component and $H_{\text {in }}$ to be those nodes with a giant out-component in $\mathcal{E}$. We define $H_{\text {scc }}=H_{\text {in }} \cap H_{\text {out }}$. The intersection $H_{\text {scc }}$ will almost surely be a strongly connected component. Here $H_{\text {in }}$ is the in-component of $H_{\text {scc }}$ and $H_{\text {out }}$ is its out-component. In general, infection of any $u \in H_{\text {in }}$ results in infection of all nodes in $H_{\text {out }}$ and occasionally a few other nodes (if $\left.u \notin H_{\mathrm{scc}}\right)$. We define such an outbreak to be an epidemic. If $u \notin H_{\text {in }}$ then a small self-limiting outbreak occurs.

For large values of $N=|G|$, the probability of an epidemic is given by $y=\mathrm{E}\left[\left|H_{\text {in }}\right|\right] / N$ and the expected fraction infected in an epidemic is given by $\mathcal{A}=\mathrm{E}\left[\left|H_{\text {out }}\right|\right] / N+\mathcal{O}(\log N / N)$. As $N$ grows, $\left|H_{\text {out }}\right| / N$ approaches $\mathrm{E}\left[\left|H_{\text {out }}\right|\right] / N$, and so the size of a single epidemic in a large population closely approximates the expected size of epidemics (note that if we include nonepidemic outbreaks in the average, this does not hold).

If the directions of arrows in the EPNs are reversed then $H_{\text {in }}$ and $H_{\text {out }}$ interchange roles. Consequently, replacing $T_{u v}=T\left(\mathcal{I}_{u}, \wp_{v}\right)$ with $\hat{T}_{u v}=T\left(\mathcal{I}_{v}, \wp_{u}\right)$ interchanges the size and probability. As such, results derived for the probability of an epidemic also apply to the size. 


\subsection{Large networks}

The results we derive will be appropriate in the limit of 'large networks'. However, in practice, we are usually interested in a single given network. Unfortunately, $|G| \rightarrow \infty$ is a vague concept when we are given a single, finite network. There are many ways to increase its size, with different impacts on epidemics. In this subsection we define what is meant by $|G| \rightarrow \infty$ in a way that allows us to produce rigorous results.

Consider a sequence of networks $\left\{G_{n}\right\}$ which satisfy $\left|G_{n}\right| \rightarrow \infty$ as $n \rightarrow \infty$. We define an open ball $B_{d}(u)$ to be a network centered at a node $u$ such that all nodes $v \in B_{d}(u)$ are at most a distance $d$ from $u$. Given a network $G$, we define $\mathrm{P}_{G}\left(B_{d}(u)\right)$ to be the probability that if we choose a node $\hat{u}$ randomly from $G$ then the set of nodes of distance at most $d$ from $\hat{u}$ is isomorphic to $B_{d}(u)$ (with the isomorphism mapping $\hat{u}$ to $u$ ).

We define sequential convergence of local statistics to mean that, given any $d$ and $B_{d}(u)$, $\mathrm{P}_{G_{n}}\left(B_{d}(u)\right)=\mathrm{P}_{G_{d}}\left(B_{d}(u)\right)$ for all $n \geq d$. For the results developed later, all that is strictly needed is that $\mathrm{P}_{G_{n}}\left(B_{d}(u)\right)$ converges as $n \rightarrow \infty$, but the stronger statement that, for $n \geq d$, they do not change makes the proofs simpler. This means that, for large enough $n$, networks have the same 'small-scale' structure, and the size of what is considered 'small-scale' increases with $|G|$. We restrict our attention to sequences which have sequential convergence of local statistics.

For a given EPN, we define $H_{\text {in }}(d)$ and $H_{\text {out }}(d)$ to be the set of nodes from which a path of length (at least) $d$ begins or ends, respectively. At large $d$, these will correspond to the $H_{\text {in }}$ and $H_{\text {out }}$ described earlier. We define $\mathcal{Y}_{d}(G)$ and $\mathcal{A}_{d}(G)$ to be the probability that a randomly chosen node from $G$ is in $H_{\text {in }}(d)$ and $H_{\text {out }}(d)$, respectively. Sequential convergence means that $\mathcal{y}_{d}\left(G_{n}\right)=\mathcal{y}_{d}\left(G_{d}\right)$ and $\mathcal{A}_{d}\left(G_{n}\right)=\mathcal{A}_{d}\left(G_{d}\right)$ for $n \geq d$. We finally define

$$
\begin{aligned}
& y=\lim _{d \rightarrow \infty} y_{d}\left(G_{d}\right), \\
& \mathcal{A}=\lim _{d \rightarrow \infty} \mathcal{A}_{d}\left(G_{d}\right) .
\end{aligned}
$$

Here $\mathcal{y}$ measures the probability of an epidemic and $\mathcal{A}$ measures the fraction infected.

We will prove our results in the limit $n \rightarrow \infty$ by showing that $H_{\text {in }}(d)$ and $H_{\text {out }}(d)$ for a given $G_{n}$ are maximal or minimal under different conditions. This means that our results are generally true for arbitrary finite networks. The reason we use the large $n$ limit is because, for networks which are small, it is unclear what constitutes a giant component in an EPN, or similarly, for a network with some unusual structure on a size comparable to the network size (for example a network made up of a few disconnected components), a giant component may not be uniquely defined. Using the large $n$ limit avoids these problems. We could avoid the need for a limit by instead assuming the existence of a giant strongly connected component in the EPN and showing that the same conditions maximize or minimize the probability a node is in the in- or out-component of this giant strongly connected component.

\section{Bounds in general networks}

We begin by considering the spread of infectious diseases on arbitrary networks. We begin with a simple lemma which we will need in this section and the next.

Lemma 1. (Edge reversal.) Given $T_{u v}=T\left(\mathcal{I}_{u}, \wp_{v}\right)$, if we interchange the roles of infectiousness and susceptibility so that $T_{u v}=T\left(\mathcal{I}_{v}, \wp_{u}\right)$ for all edges, then $\mathcal{Y}$ and $\mathcal{A}$ interchange roles. 
Proof. If we replace $T_{u v}=T\left(\mathcal{I}_{u}, \wp_{v}\right)$ with $\hat{T}_{u v}=T\left(\mathcal{I}_{v}, \wp_{u}\right)$ then the new EPNs correspond to reversing the direction of edges in the original EPNs. Since reversing the direction of edges in an EPN interchanges $H_{\text {in }}(d)$ and $H_{\text {out }}(d)$, this interchanges $\mathcal{y}$ and $\mathcal{A}$, and completes the proof.

We now make a simplifying assumption which we will need for networks with short cycles.

Assumption 1. (Ordering assumption.) If $T\left(\mathcal{I}_{1}, \S_{1}\right)>T\left(\mathcal{I}_{2}, \wp_{1}\right)$ for any $\S_{1}$ then $T\left(\mathcal{I}_{1}, \S\right) \geq$ $T\left(I_{2}, 8\right)$ for all \&. Furthermore, strict inequality occurs for a set of positive measure. Similarly, if $T\left(\mathcal{I}_{1}, \wp_{1}\right)>T\left(\mathcal{I}_{1}, \wp_{2}\right)$ for any $\mathcal{I}_{1}$ then $T\left(\mathcal{I}, \wp_{1}\right) \geq T\left(\mathcal{I}, \wp_{2}\right)$ for all $\mathcal{I}$ with strict inequality for a set of positive measure.

The ordering assumption is a statement about the functional form of $T(\mathcal{L}, \mathcal{\&})$. It places no restrictions on the network. The assumption holds for (1), but as noted earlier there are many scenarios where it fails.

The ordering assumption implies that $T_{\text {out }}(\mathcal{L})$ and $T_{\text {in }}(\&)$ are invertible mappings. It also allows us to assume that $\mathcal{I}$ is a scalar quantity ordered such that

$$
I_{u} \geq \mathcal{I}_{u^{\prime}} \Longleftrightarrow T\left(I_{u}, \delta\right) \geq T\left(\mathcal{I}_{u^{\prime}}, \delta\right) \text { for all } \delta \quad \Longleftrightarrow \quad T_{\text {out }}(u) \geq T_{\text {out }}\left(u^{\prime}\right)
$$

and, furthermore, that $\tau_{u}>\mathcal{I}_{u^{\prime}}$ is equivalent to $T_{\text {out }}(u)>T_{\text {out }}\left(u^{\prime}\right)$. We may make similar conclusions about $\delta$. There will be more than one way to represent $\mathcal{L}$ or $\delta$ as scalars. It will frequently (but not always) be convenient to identify $\mathcal{I}$ with $T_{\text {out }}(\mathcal{I})$ and $\delta$ with $T_{\text {in }}(\&)$.

Previous work by Kuulasmaa [16] considered the spread of infectious diseases on networks for which the only heterogeneity came from variation in the duration of the infection. Hence, all nodes have the same $T_{\mathrm{in}}$, and variation occurs only in $T_{\text {out }}$. This model satisfies the ordering assumption. In this section we generalize the results of [16] by allowing $T_{\text {in }}$ and $T_{\text {out }}$ to be heterogeneous simultaneously.

We will drop the ordering assumption in Section 4, where we consider networks with no short cycles. Even in this section, many of the results hold without the ordering assumption, but the proofs are less clean. The assumption is only strictly needed for Theorems 1, 3, and 4, below.

We are now ready to show that increased heterogeneity generally decreases the size and probability of epidemics. We show that, for a given $Q_{\text {in }}$ or $Q_{\text {out }}$, both $\mathcal{y}$ and $\mathcal{A}$ are maximal when $T_{\text {out }}$ or, respectively, $T_{\text {in }}$ is homogeneous. They are minimal when the variance of $T_{\text {out }}$ or $T_{\text {in }}$ is maximal subject to the constraint of $Q_{\text {in }}$ or, respectively, $Q_{\text {out }}$.

We can also derive conditions for a global upper bound on $\mathcal{y}$ and $\mathcal{A}$. The upper bounds occur when $T_{u v}=\langle T\rangle$ for all neighbors $u$ and $v$. We hypothesize a lower bound, but cannot prove it in networks with short cycles.

To make the notation cleaner in the following lemma, we identify $\&$ with $T_{\text {in }}$ and so we may use $T\left(\mathcal{L}, T_{\text {in }}\right)$ in place of $T(\mathcal{L}, \S)$.

Lemma 2. Assume a sequence of networks $\left\{G_{n}\right\}$ with sequential convergence of local statistics and a susceptibility distribution $Q_{\mathrm{in}}\left(T_{\mathrm{in}}\right)$. Assume that the ordering assumption holds and consider a distribution of infectiousness $P_{1}(\mathcal{I})$ with transmissibility given by $T_{1}\left(\mathcal{I}, T_{\mathrm{in}}\right)$, that is consistent with $Q_{\mathrm{in}}$. Let $\phi_{\mathrm{in}, 1}(V, \vec{\jmath})$ be as in (2). Let $\mathcal{A}_{1}$ and $\mathcal{y}_{1}$ be the corresponding attack rate and epidemic probability, respectively. Similarly, choose another $P_{2}(\mathcal{I}), T_{2}\left(\mathcal{I}, T_{\text {in }}\right)$ with 
corresponding $\mathcal{A}_{2}, y_{2}$, and $\phi_{2}(V, \vec{\jmath})$. Assume that $\phi_{\mathrm{in}, 1}(V, \vec{\jmath}) \leq \phi_{\mathrm{in}, 2}(V, \vec{\jmath})$ for all $V$ and $\vec{\jmath}$. Then $\mathcal{A}_{1} \geq \mathcal{A}_{2}$ and $y_{1} \geq \mathcal{y}_{2}$.

Proof. Let $d \geq 0$ be given.

Take $G_{n}, n \geq d$. We will show that a node in an EPN created from $G_{n}$ using the first distribution is more likely to be in $H_{\text {out }}(d)$ than a node in an EPN created using the second distribution.

Choose any node $u$ from $G_{n}$. Partition the nodes of $G_{n}$ into disjoint sets $\{u\}, U_{1}$, and $U_{2}$. To the nodes in $U_{1}$ we assign $\mathcal{I}$ from $P_{1}(\mathcal{I})$ and to the nodes in $U_{2}$ we assign $\mathcal{I}$ from $P_{2}(\mathcal{I})$. We assign $T_{\text {in }}$ to all nodes from $Q_{\text {in }}\left(T_{\text {in }}\right)$. We will consider the effects of adding $u$ to $U_{1}$ versus adding it to $U_{2}$.

Consider a partial EPN $\&$ created by assigning edges $(w, v)$ from all $w \neq u$, using $T_{1}\left(\mathcal{I}_{w}, T_{i}(v)\right)$ if $w \in U_{1}$ and $T_{2}\left(\mathcal{I}_{w}, T_{i}(v)\right)$ if $w \in U_{2}$. Now consider an arbitrary node $u^{\prime}$ (which may be $u$ ) which is not already in $H_{\text {in }}(d)$, but which would join $H_{\text {in }}(d)$ if the appropriate edges were added from $u$. Let $V$ be the set of neighbors $v$ of $u$ for which adding the edge $(u, v)$ would allow a path from $u^{\prime}$ to $u$ to be extended to a path of length $d$.

We consider extensions of $\varepsilon$ formed by placing $u$ into $U_{1}$ or $U_{2}$. The probability that $u^{\prime}$ would be in $H_{\text {in }}(d)$ in the extended EPN is equal to the probability that $u$ has at least one edge to some node in $V$. This probability is at least as high if $u \in U_{1}$ as if $u \in U_{2}$ by our assumption $\phi_{\text {in, } 1}(V, \vec{S}) \leq \phi_{\text {in, } 2}(V, \vec{S})$. Consequently, the probability of $u^{\prime}$ being in $H_{\text {in }}(d)$ is maximal if $u \in U_{1}$. Induction on $\left|U_{1}\right|$ shows that $\mathcal{y}_{d}\left(G_{n}\right)$ is largest if all nodes are in $U_{1}$.

We now show that $u \in U_{1}$ increases $\mathcal{A}_{d}$ compared with $u \in U_{2}$. We can prove that placing $u$ in $U_{1}$ versus $U_{2}$ can only increase the probability of a node being at the end of a length $d$ path. The proof proceeds largely as above. Consider the same partial EPN $\&$ defined above. Let $u^{\prime}$ be a node which is not in $H_{\text {out }}(d)$ but would be if an edge from $u$ to any $v \in V$ were added (note that $u \neq u^{\prime}$ ). The probability that $u^{\prime}$ will be in $H_{\text {out }}(d)$ is $\phi_{\text {in }, 1}(V, \vec{s})$ or $\phi_{\text {in }, 2}(V, \vec{s})$ depending on whether $u$ is assigned $\mathcal{I}$ from $P_{1}$ or $P_{2}$. Because $\phi_{\text {in, } 1}(V, \vec{\jmath}) \leq \phi_{\text {in }, 2}(V, \vec{\jmath})$, it follows that $\mathcal{A}_{d}$ is largest if $u \in U_{1}$. Induction on $\left|U_{1}\right|$ shows that $\mathscr{A}_{d}\left(G_{n}\right)$ is maximal if all nodes are in $U_{1}$.

Taking $d \rightarrow \infty$, it then follows that $\mathcal{Y}$ and $\mathcal{A}$ are maximal if all nodes are in $U_{1}$, completing the proof.

We begin by showing that, for a fixed distribution of in-transmissibility, the size and probability are largest when the out-transmissibility is homogeneous.

Theorem 1. Let $Q_{\mathrm{in}}\left(T_{\mathrm{in}}\right)$ be given. Assume that the ordering assumption holds and that $\left\{G_{n}\right\}$ satisfies sequential convergence of statistics. Set $\wp_{v}=T_{\mathrm{in}}(v)$. Then $y$ and $\mathcal{A}$ are maximized when $T\left(\mathcal{I}, T_{\text {in }}\right)=T_{\text {in }}$.

Proof. By the ordering assumption, we may take $\mathcal{I}$ to be scalar with $\tau_{1}>I_{2}$ if and only if $T_{\text {out }}\left(\mathcal{I}_{1}\right)>T_{\text {out }}\left(\mathcal{L}_{2}\right)$. This allows us to use Chebyshev's 'other' inequality [15]: if $h_{1}$ and $h_{2}$ are decreasing functions of $x$ and $p$ is a probability density function,

$$
\int h_{1}(x) h_{2}(x) p(x) \mathrm{d} x \geq\left(\int h_{1}(x) p(x) \mathrm{d} x\right)\left(\int h_{2}(x) p(x) \mathrm{d} x\right) .
$$

By induction, $\int\left(\prod h_{j}(x)\right) p(x) \mathrm{d} x \geq \prod \int h_{j}(x) p(x) \mathrm{d} x$ for any number of decreasing functions $h_{j}$. 
Applying this to the decreasing function $h_{j}(\mathcal{I})=1-T\left(\mathcal{L}, T_{\text {in }}\left(v_{j}\right)\right)$, we have

$$
\begin{aligned}
\phi_{\text {in }}(V, \vec{\jmath}) & =\int\left(\prod_{v \in V} h_{j}(\mathcal{I})\right) P(\mathcal{I}) \mathrm{d} \mathcal{I} \\
& \geq \prod_{v \in V} 1-T_{\text {in }}(v),
\end{aligned}
$$

with equality if $T\left(\mathcal{I}, T_{\text {in }}\right)=T_{\text {in }}$. Thus, by Lemma $2, \mathcal{A}$ and $\mathcal{y}$ are maximal, completing the proof.

We have proven the upper bounds given that $Q_{\text {in }}\left(T_{\text {in }}\right)$ occur when $T_{\text {out }}$ is homogeneous. We now show the lower bounds occur when $T_{\text {out }}$ is maximally heterogeneous. Because of the ordering assumption, we may take $\ell_{v}=T_{\text {in }}(v)$.

Theorem 2. Let $Q_{\text {in }}\left(T_{\text {in }}\right)$ be given. Assume that the ordering assumption holds and that $\left\{G_{n}\right\}$ satisfies sequential convergence of statistics. Take $\tau$ to be chosen uniformly from $[0,1]$. Setting

$$
T\left(\mathcal{I}, T_{\text {in }}\right)= \begin{cases}0, & T_{\text {in }}<\mathcal{I}, \\ 1, & T_{\text {in }}>\mathcal{I},\end{cases}
$$

minimizes $\mathcal{y}$ and $\mathcal{A}$.

Proof. Given (5), we have $\phi_{\text {in }}(V, \vec{s})=\min _{v \in V}\left\{1-T_{\text {in }}(v)\right\}$.

We need to prove that, for any arbitrary transmission function $\hat{T}\left(\mathcal{L}, T_{\text {in }}\right)$ satisfying the ordering assumption and consistent with $T_{\text {in }}, \phi_{\text {in }}(V, \vec{f}) \leq \min _{v \in V}\left\{1-T_{\text {in }}(v)\right\}$. To do this, let $\hat{T}$ be given, let $T_{\text {in }}$ be assigned to $v_{1}, \ldots, v_{n}$, and assume that $v_{1}, \ldots, v_{n}$ are ordered such that $T_{\text {in }}\left(v_{1}\right) \geq T_{\text {in }}\left(v_{2}\right) \geq \cdots \geq T_{\text {in }}\left(v_{n}\right)$. Then

$$
\begin{aligned}
\phi_{\text {in }}(V, \overrightarrow{\mathcal{S}}) & =\int \prod_{j=1}^{n}\left(1-\hat{T}\left(\mathcal{I}, T_{\text {in }}\left(v_{j}\right)\right)\right) P(\mathcal{I}) \mathrm{d} \mathcal{I} \\
& \leq \int\left(1-\hat{T}\left(\mathcal{I}, T_{\text {in }}\left(v_{1}\right)\right)\right) P(\mathcal{I}) \mathrm{d} \mathcal{I} \\
& \leq 1-T_{\text {in }}\left(v_{1}\right) .
\end{aligned}
$$

This shows that, for any $\hat{T}, \phi_{\text {in }}(V, \vec{f})$ is at most the value it takes for (5). Thus, Lemma 2 shows that $\mathcal{y}$ and $\mathcal{A}$ are minimal, completing the proof.

We derived the results above with fixed $Q_{\text {in }}$. Lemma 1 shows that the equivalent results must hold for $Q_{\text {out }}$.

Theorem 3. Let $Q_{\text {out }}\left(T_{\text {out }}\right)$ be given. Assume that the ordering assumption holds and that $\left\{G_{n}\right\}$ satisfies sequential convergence of statistics. Set $\tau_{u}=T_{\text {out }}(u)$.

- If $T\left(T_{\text {out }}, \&\right)=T_{\text {out }}$ then $y$ and $\mathcal{A}$ are maximized.

- If $\&$ is chosen uniformly in $[0,1]$ and

$$
T\left(T_{\text {out }}, \&\right)= \begin{cases}0, & T_{\text {out }}<\&, \\ 1, & T_{\text {out }}>s,\end{cases}
$$

then $y$ and $\mathcal{A}$ are minimized. 
Proof. This follows immediately from Lemma 1 with Theorems 1 and 2.

We now give a global upper bound for both $\mathcal{y}$ and $\mathcal{A}$.

Theorem 4. Let $\langle T\rangle$ be given. Under the ordering assumption with sequential convergence of statistics for $\left\{G_{n}\right\}$, the maximum of $\mathcal{y}$ and $\mathcal{A}$ occur when $T_{u v}=\langle T\rangle$ for all neighboring nodes.

Proof. Consider a $P(\mathcal{I}), P(\&)$, and $T(\mathcal{I}, \S)$ which yield a global maximum for either $\mathcal{Y}$ or $\mathcal{A}$. If $T_{\text {in }}$ is not homogeneous then we can find a new infection process which preserves the same $Q_{\text {out }}\left(T_{\text {out }}\right)$ with homogeneous $T_{\text {in }}$ which can only increase $\mathcal{y}$ or $\mathcal{A}$. A repeated application preserving the new homogeneous in-transmissibility, but now making $T_{\text {out }}$ also homogeneous again can only increase $\mathcal{y}$ or $\mathcal{A}$. Then $T_{\text {out }}$ and $T_{\text {in }}$ are then homogeneous. This completes the proof.

We finish with a conjecture about global lower bounds.

Conjecture 1. Under the ordering assumption with sequential convergence of statistics for $\left\{G_{n}\right\}$, the minimum of $\mathcal{y}$ occurs when $Q_{\text {out }}\left(T_{\text {out }}\right)=\langle T\rangle \delta\left(T_{\text {out }}-1\right)+(1-\langle T\rangle) \delta\left(T_{\text {out }}\right)$.

The minimum of $\mathcal{A}$ occurs when $Q_{\mathrm{in}}\left(T_{\mathrm{in}}\right)=\langle T\rangle \delta\left(T_{\mathrm{in}}-1\right)+(1-\langle T\rangle) \delta\left(T_{\mathrm{in}}\right)$.

Note that if $Q_{\text {out }}\left(T_{\text {out }}\right)=\langle T\rangle \delta\left(T_{\text {out }}-1\right)+(1-\langle T\rangle) \delta\left(T_{\text {out }}\right)$ then $Q_{\text {in }}\left(T_{\text {in }}\right)=\delta\left(T_{\text {in }}-\langle T\rangle\right)$ is homogeneous.

\subsection{Discussion}

The results of Section 3 have focused on extending earlier results of Kuulasmaa [16] who considered a population with homogeneous susceptibility and heterogeneities in infectiousness due entirely to variation in the duration of the infection. We have extended these results to cover a wide range of heterogeneities in infectiousness and susceptibility (simultaneously), under the assumption that infectiousness and susceptibility are assigned independently. In order to extend the proof used by Kuulasmaa, we have been forced to make the ordering assumption, which effectively means that if we order people by how infectious they would be to one test-susceptible individual, the order is the same as we would find for another test-susceptible individual. We do not have any counterexamples to these theorems in the case where the ordering assumption fails, and so it is not clear that it is needed. In Section 4 we will see that similar results hold in unclustered networks without needing the ordering assumption.

Our results show that in general increasing the heterogeneity of the population is useful for either decreasing the size or decreasing the probability that an epidemic occurs. Given $Q_{\text {in }}$ or $Q_{\text {out }}$, both $\mathcal{Y}$ and $\mathcal{A}$ are maximized if $T_{\text {out }}$ or, respectively, $T_{\text {in }}$ is homogeneous and minimized if it is maximally heterogeneous. Similarly, given just $\langle T\rangle$, we find that the global maxima of $\mathcal{y}$ and $\mathcal{A}$ occur when $T=\langle T\rangle$. Perhaps surprisingly, the conditions leading to upper and lower bounds are independent of the network, though the size of the variation between these bounds is network dependent.

Although we can prove lower bounds given $Q_{\text {in }}$ (or $Q_{\text {out }}$ ), we cannot prove global lower bounds given $\langle T\rangle$. We hypothesize that the global lower bound for $y$ occurs when $Q_{\text {out }}$ is maximally heterogeneous and the global lower bound for $\mathcal{A}$ occurs when $Q_{\text {in }}$ is maximally heterogeneous. In the next section we will see that these are the lower bounds for an unclustered population. However, we have not found a rigorous proof for general networks. In the proof of the upper bound, we took a given $Q_{\text {out }}$ and found $Q_{\text {in }}$ that maximizes $y$ and $\mathcal{A}$. We then held that $Q_{\text {in }}$ fixed and found $Q_{\text {out }}$ to maximize $y$ and $\mathcal{A}$, arriving at the upper bound. However, applying a similar technique to the lower bound fails because given any $Q_{\text {out }}$, if we find a 
minimizing $Q_{\text {in }}$, attempting to then minimize with $Q_{\text {in }}$ fixed simply returns the original $Q_{\text {out }}$. The difficulty results from the fact that increasing heterogeneity in $T_{\text {out }}$ restricts the amount of heterogeneity in $T_{\text {in }}$ and vice versa.

\section{Bounds in unclustered networks}

Most studies of infectious diseases spreading on networks have been made for networks for which the effect of short cycles may be neglected [25]. These investigations have generally used Molloy-Reed networks [23] (also known as the configuration model [26]). The theory we develop here applies to these networks, but also to more general networks which may have degree-degree correlations, or even longer range correlations.

When we study networks with no short cycles, we are able to prove stronger results and abandon the ordering assumption. We find that $y$ depends on the network and $Q_{\text {out }}\left(T_{\text {out }}\right)$ only, while $\mathcal{A}$ depends on the network and $Q_{\text {in }}\left(T_{\text {in }}\right)$ only. We can prove global upper and (unlike in the general case) lower bounds on $\mathcal{Y}$ and $\mathcal{A}$.

Assumption 2. (Unclustered assumption.) Given a sequence of networks $\left\{G_{n}\right\}$, we assume that $G_{n}$ has girth greater than $2 n$.

This assumption means that $B_{d}(u)$ chosen from any $G_{n}$ with $n \geq d$ must be cycle free. In particular, there is no alternate path between a node and a neighbor. It was this complication that forced the use of the ordering assumption earlier, and since the complication no longer exists, we drop the ordering assumption. The unclustered assumption will also allow us to use $\psi_{\text {in }}(V)$ rather than $\phi_{\text {in }}(V, \vec{S})$. Thus, we only require the marginal probability of the set of nodes $V$ not to be infected to satisfy an inequality, rather than the inequality be satisfied for every possible set of susceptibilities. We must bear in mind that knowing $T_{\text {in }}$ or $T_{\text {out }}$ no longer uniquely determines $\tau$ or $\delta$.

Lemma 3. Let the sequence $\left\{G_{n}\right\}$ satisfy the unclustered assumption with sequential convergence of statistics. Take $P_{1}(\mathcal{I}), P_{1}(\S)$, and $T_{1}(\mathcal{I}, \S)$. Let $\psi_{\mathrm{in}, 1}(V)$ be as in (4). Similarly, take $P_{2}(\mathcal{I}), P_{2}(\&)$, and $T_{2}(\mathcal{I}, \&)$ with corresponding $\psi_{\mathrm{in}, 2}(V)$. If $\psi_{\mathrm{in}, 1}(V) \leq \psi_{\mathrm{in}, 2}(V)$ then $y_{1} \geq y_{2}$.

Proof. This proof is similar to that of Lemma 2.

Let $d \geq 0$ be given. Take $G_{n}, n \geq d$. Choose a node $u$ from $G_{n}$ and partition the nodes of $G_{n}$ into $\{u\}, U_{1}$, and $U_{2}$. To the nodes in $U_{1}$ we assign $\mathcal{I}$ from $P_{1}(\mathcal{I})$ and to the nodes of $U_{2}$ we assign $\mathcal{I}$ from $P_{2}(\mathcal{L})$. To each node $w$ (including $u$ ) we assign two susceptibilities, $\wp_{w, 1}$ and $\S_{w, 2}$, such that $\S_{w, 1}$ comes from $P_{1}(\&)$ and $\S_{w, 2}$ comes from $P_{2}(\&)$.

We create a partial EPN $\&$ as follows. For each $v \in U_{1}$, we assign edges $(v, w)$ using $T_{1}\left(\mathcal{I}_{v}, \wp_{w, 1}\right)$ and, for $v \in U_{2}$, we assign them using $T_{2}\left(\mathcal{I}_{v}, \wp_{w, 2}\right)$. We do not yet assign edges from $u$ (but edges may point to $u$ ). Consider any $u^{\prime}$ not in $H_{\text {in }}(d)$ which would join $H_{\text {in }}(d)$ if an edge was added from $u$ to any $v \in V$. By assumption, $\psi_{\mathrm{in}, 1}(V) \leq \psi_{\mathrm{in}, 2}(V)$ and so the probability is greatest if $\mathcal{I}_{u}$ is chosen from $P_{1}(\mathcal{I})$. It follows that $\mathcal{y}_{1} \geq \mathcal{Y}_{2}$. This completes the proof.

This proof can be modified to work on clustered networks without the ordering assumption, so Lemma 2 does not require the ordering assumption. However, the proof is more technical and provides little additional insight, particularly because the main results following from Lemma 2 do require the ordering assumption. 
Theorem 5. Let the sequence $\left\{G_{n}\right\}$ satisfy the unclustered assumption with sequential convergence of local statistics. Let $Q_{\mathrm{in}}\left(T_{\mathrm{in}}\right)$ be fixed. Then $\mathcal{A}$ is fixed.

Proof. We follow the technique used to prove that $\mathcal{A}$ is larger for one distribution than the other in Lemma 2. However, in following that proof, the lack of clustering means that $|V|=1$. Since, for any distribution, $\psi(V)=1-\langle T\rangle$ when $|V|=1$, all distributions must give the same $\mathcal{A}$, completing the proof.

Theorem 6. If the assumptions of Theorem 5 hold, except that $Q_{\text {out }}$ is fixed rather than $Q_{\text {in }}$, then $y$ is fixed.

Proof. This follows immediately from Lemma 1 and Theorem 5.

Theorem 7. Let $Q_{\text {in }}$ be given. Assume that $\left\{G_{n}\right\}$ satisfies the unclustered assumption with sequential convergence of statistics. Then $y$ is maximized when $T(\mathcal{L}, \&)=T_{\text {in }}(\&)$.

Although this result is analogous to Theorem 1, the proof is fundamentally altered because we no longer have the ordering assumption.

Proof of Theorem 7. We first note that if $T(\mathcal{L}, \&)=T_{\text {in }}(\&)$ then $T_{\text {out }}=\langle T\rangle$ for all nodes.

Now consider an arbitrary function $T(\mathcal{L}, \delta)$ with $P(\mathcal{L})$ and $P(\delta)$ to satisfy $Q_{\text {in }}\left(T_{\text {in }}\right)$. The function (1- $\left.T_{\text {out }}\right)^{|V|}$ in (4) is convex so, by Jensen's inequality, $\psi_{\text {in }}$ is minimized by $T_{\text {out }}=\langle T\rangle$. Lemma 3 completes the proof.

Theorem 8. Let $Q_{\text {in }}$ be given. Assume that $\left\{G_{n}\right\}$ satisfies the unclustered assumption with sequential convergence of statistics. Then $\mathcal{y}$ is minimized when $\mathcal{I}$ is chosen uniformly from $[0,1]$ and

$$
T(\mathcal{I}, \S)= \begin{cases}0, & \mathcal{I}>T_{\text {in }}(\&), \\ 1, & \mathcal{I}<T_{\text {in }}(\wp) .\end{cases}
$$

Proof. Following the proof of Theorem 2, we may show that $\phi_{\text {in }}$ is maximized (subject to $Q_{\text {in }}$ ) exactly when these assumptions hold. Thus, from (3), $\psi_{\text {in }}$ is also maximized when these assumptions hold. Lemma 3 completes the proof.

Theorem 9. Let $Q_{\text {out }}$ be given. Assume that $\left\{G_{n}\right\}$ satisfies the unclustered assumption with sequential convergence of statistics.

- A is maximized when $T(\mathcal{I}, \mathcal{S})=T_{\text {out }}(\mathcal{I})$.

- $\mathcal{A}$ is minimized when 8 is chosen uniformly from $[0,1]$ and

$$
T(\mathcal{I}, \&)= \begin{cases}0, & \&>T_{\mathrm{out}}(\mathcal{I}) \\ 1, & \&<T_{\mathrm{out}}(\mathcal{I})\end{cases}
$$

Proof. This follows from Theorems 7 and 8 with Lemma 1.

Before proving our final result, we introduce a lemma.

Lemma 4. Let $f(x)$ be a convex function on $[0,1]$, and let $\rho(x)$ be a probability density function on $[0,1]$, with expected value $\rho_{0}$. Then

$$
\int f(x) \rho(x) \mathrm{d} x \leq\left(1-\rho_{0}\right) f(0)+\rho_{0} f(1) .
$$


Proof. The definition of convexity gives $f(x) \leq(1-x) f(0)+x f(1)$. Thus,

$$
\int f(x) \rho(x) \mathrm{d} x \leq \int((1-x) f(0) \rho(x)+x f(1) \rho(x)) \mathrm{d} x \leq\left(1-\rho_{0}\right) f(0)+\rho_{0} f(1) .
$$

Theorem 10. Let $\left\{G_{n}\right\}$ be a sequence of networks satisfying the unclustered assumption with sequential convergence of statistics. Assume that $\langle T\rangle$ is given.

- The global upper bound for both $\mathcal{y}$ and $\mathcal{A}$ occurs when $T_{u v}=\langle T\rangle$ for all pairs of neighbors.

- The global lower bound for $y$ occurs when $Q_{\text {out }}\left(T_{\text {out }}\right)=\langle T\rangle \delta\left(T_{\text {out }}-1\right)+(1-$ $\langle T\rangle) \delta\left(T_{\text {out }}\right)$.

- The global lower bound for $\mathcal{A}$ occurs when $Q_{\text {in }}\left(T_{\text {in }}\right)=\langle T\rangle \delta\left(T_{\text {in }}-1\right)+(1-\langle T\rangle) \delta\left(T_{\text {in }}\right)$.

Proof. The proof of the upper bound is identical to that of Theorem 4.

We prove the lower bound for $\mathcal{y}$. The lower bound for $\mathcal{A}$ follows from Lemma 1.

We have $\psi_{\text {in }}(V)=\int\left(1-T_{\text {out }}\right)^{|V|} Q_{\text {out }}\left(T_{\text {out }}\right) \mathrm{d} T_{\text {out }}$. We now seek to find $Q_{\text {out }}$, which maximizes $\psi_{\text {in }}$, in order to apply Lemma 3.

Since $\left(1-T_{\text {out }}\right)^{|V|}$ is a convex function, we may apply Lemma 4 with $Q_{\text {out }}$ playing the role of $\rho$. The maximum occurs when $Q_{\text {out }}\left(T_{\text {out }}\right)=\langle T\rangle \delta\left(T_{\text {out }}-1\right)+(1-\langle T\rangle) \delta\left(T_{\text {out }}\right)$, and so Lemma 3 completes the proof.

Although the upper bound for both $\mathcal{Y}$ and $\mathcal{A}$ occurs when $T_{u v}=\langle T\rangle$ for all pairs, our earlier results show that, for unclustered networks, $y$ depends only on $Q_{\text {out }}\left(T_{\text {out }}\right)$ and the network, and so as long as $T_{\text {out }}(u)=\langle T\rangle$ for all nodes $u$, we achieve the upper bound on $y$ (but not on $\mathcal{A})$. Symmetrically, if $T_{\text {in }}(v)=\langle T\rangle$ for all nodes $v$, we achieve the upper bound on $\mathcal{A}$.

Note that the lower bound for $\mathcal{A}$ requires that $T_{\text {out }}(u)=\langle T\rangle$ for all $u$, and so the population is homogeneously infectious. It follows from Theorem 8 that $y$ is then maximal. Similarly, the lower bound for $\mathcal{Y}$ requires that $\mathcal{A}$ be maximal.

\subsection{Discussion}

The results of Section 4 generalize those of [21], which considered the special case of Molloy-Reed networks. These results prove that the same scenarios give upper and lower bounds in unclustered networks with a wide range of correlations including assortative or disassortative mixing (high-degree nodes preferentially joining with high- or low-degree nodes, respectively), or longer range correlations. Although we proved these under the assumption that no short cycles exist, the results remain useful in networks with either few short cycles, or for situations in which the transmissibility is low enough that the short cycles are only rarely followed.

Because of the lack of short cycles, the ordering assumption is not needed. This means that our results apply to a much wider class of disease transmission mechanisms, but at the cost of restricting the network. Again we find that the conditions which give the upper or lower bound are network independent. The amount of variation there is between these bounds, however, is network dependent.

The main distinction from clustered networks is that $y$ depends only on the network structure and $Q_{\text {out }}\left(T_{\text {out }}\right)$. That is, $\mathcal{Y}$ is independent of $Q_{\text {in }}\left(T_{\text {in }}\right)$. Similarly, $\mathcal{A}$ depends only on the network structure and $Q_{\text {in }}\left(T_{\text {in }}\right)$. We note that unless the effect of clustering is very large, the dependence of $\mathcal{y}$ on in-transmissibility and $\mathcal{A}$ on out-transmissibility will be weak. Curiously, the global lower bound for $\mathcal{A}$ found in Theorem 10 requires that $Q_{\text {out }}\left(T_{\text {out }}\right)=\delta\left(T_{\text {out }}-\langle T\rangle\right)$, and so the 
population is homogeneously susceptible. It follows from Theorem 8 that $y$ is then maximal. Similarly, the lower bound for $\mathcal{Y}$ requires that $\mathscr{A}$ be maximal. This has important implications for policy design because strategies to reduce $T$ tend to have a heterogeneous impact on either $\delta$ or $\mathcal{L}$.

\section{Conclusions}

We have extended earlier work on the effect of heterogeneity in infectiousness on the spread of epidemics through networks. Our extensions allow for heterogeneity in susceptibility as well. Many of the results are similar. In general, we find that the size and probability of epidemics are reduced if the population is more heterogeneous. Unfortunately, increasing heterogeneity in susceptibility restricts the level of heterogeneity possible in infectiousness. In the extreme case where susceptibility is maximally heterogeneous, infectiousness must be homogeneous. Perhaps surprisingly, we have found that the distributions leading to upper and lower bounds on $\mathcal{y}$ and $\mathcal{A}$ are network independent.

Early in an outbreak, it is likely that we may gain some information about $Q_{\text {out }}\left(T_{\text {out }}\right)$. For example, in the early stages of the SARS epidemic, it was known that a number of people were highly infectious, while the rest were only mildly infectious and so $Q_{\text {out }}\left(T_{\text {out }}\right)$ was highly heterogeneous. However, there was little information on $Q_{\text {in }}$. Once given the distribution of $Q_{\text {out }}$, the results here show which distributions of $Q_{\text {in }}$ give the largest or smallest $\mathcal{y}$ and $\mathcal{A}$. Our results further suggest that the distribution of infectiousness found for SARS is consistent with a low epidemic probability. It is difficult to extrapolate from observations what the sizes would have been without the interventions put into place, but the fact that a number of isolated cases occurred throughout the world without sparking local epidemics suggest that the probability of an epidemic from each introduction was low, consistent with our predictions.

Our results further suggest that in order to prevent an epidemic, it is best to take measures that will have a heterogeneous impact on infectiousness, but in order to affect the size of an epidemic, it is best to take measures that will have a heterogeneous impact on susceptibility. In terms of actual interventions, we compared two strategies aimed at controlling a disease which is initially spreading with homogeneous $T$ : in the first we devote resources to vaccinating half of the population, while in the second we devote them to identifying and removing half of the infected population. Both strategies reduce $\langle T\rangle$ by a factor of 2 . In the first, the susceptibility is highly heterogeneous, but the probability an infected node infects a randomly chosen neighbor has simply gone down by a factor of 2 , and so it remains homogeneous. Assuming that the unclustered approximation is valid, this maximizes the impact on size, but the impact on probability is minimized. In contrast, the second strategy maximizes the impact on probability, but minimizes the impact on size.

\section{Acknowledgements}

This work was supported in part by the Division of Mathematical Modeling at the UBC CDC and by DOE at LANL under contract DE-AC52-06NA25396 and the DOE Office of ASCR program in Applied Mathematical Sciences.

\section{References}

[1] Abbey, H. (1952). An examination of the Reed-Frost theory of epidemics. Human Biol. 24, 201-233.

[2] Anderson, R. M. And May, R. M. (1991). Infectious Diseases of Humans. Oxford University Press.

[3] Andersson, H. (1999). Epidemic models and social networks. Math. Scientist 24, 128-147. 
[4] Ball, F. (1985). Deterministic and stochastic epidemics with several kinds of susceptibles. Adv. Appl. Prob. 17, $1-22$.

[5] Ball, F. ANd O'NeiLl, P. (1999). The distribution of general final state random variables for stochastic epidemic models. J. Appl. Prob. 36, 473-491.

[6] Broder, A. et al. (2000). Graph structure in the web. Comput. Networks 33, 309-320.

[7] Del Valle, S. Y., Hyman, J. M., Hethcote, H. W. and Eubank, S. G. (2007). Mixing patterns between age groups in social networks. Social Networks 29, 539-554.

[8] Eubank, S. et al. (2004). Modelling disease outbreaks in realistic urban social networks. Nature 429, $180-184$.

[9] Hastings, M. B. (2006). Systematic series expansions for processes on networks. Phys. Rev. Lett. 96, 148701.

[10] Keeling, M. J. (2005). The implications of network structure for epidemic dynamics. Theoret. Pop. Biol. 67, $1-8$.

[11] Keeling, M. J. and Eames, K. T. D. (2005). Networks and epidemic models. J. R. Soc. Interf. 2, 295-307.

[12] Kenah, E. And Robins, J. M. (2007). Network-based analysis of stochastic SIR epidemic models with random and proportionate mixing. J. Theoret. Biol. 249, 706-722.

[13] Kenah, E. And Robins, J. M. (2007). Second look at the spread of epidemics on networks. Phys. Rev. E 76, 036113.

[14] Kermack, W. O. and McKendrick, A. G. (1927). A contribution to the mathematical theory of epidemics. Proc. R. Soc. London Ser. A 115, 700-721.

[15] Kingman, J. F. C. (1978). Uses of exchangeability. Ann. Prob. 6, 183-197.

[16] KuulasmaA, K. (1982). The spatial general epidemic and locally dependent random graphs. J. Appl. Prob. 19, 745-758.

[17] KuUlasmaa, K. and Zachary, S. (1984). On spatial general epidemics and bond percolation processes. J. Appl. Prob. 21, 911-914.

[18] Madar, N. et al. (2004). Immunization and epidemic dynamics in complex networks. Europ. Phys. J. B 38, 269-276.

[19] Meyers, L. A. (2007). Contact network epidemiology: bond percolation applied to infectious disease prediction and control. Bull. Amer. Math. Soc. 44, 63-86.

[20] Meyers, L. A., Newman, M. and Pourbohloul, B. (2006). Predicting epidemics on directed contact networks. J. Theoret. Biol. 240, 400-418.

[21] Miller, J. C. (2007). Epidemic size and probability in populations with heterogeneous infectivity and susceptibility. Phys. Rev. E 76, 010101.

[22] Mollison, D. (1977). Spatial contact models for ecological and epidemic spread. J. R. Statist. Soc. Ser. B 39, 283-326.

[23] Molloy, M. And Reed, B. (1995). A critical point for random graphs with a given degree sequence. Random Structures Algorithms 6, 161-179.

[24] Neal, P. (2007). Copuling of two SIR epidemic models with variable susceptibilities and infectivities. J. Appl. Prob. 44, 41-57.

[25] Newman, M. E. J. (2002). Spread of epidemic disease on networks. Phys. Rev. E 66, 16128.

[26] Newman, M. E. J. (2003). The structure and function of complex networks. SIAM Rev. 45, 167-256.

[27] Pastor-Satorras, R. and Vespignani, A. (2001). Epidemic spreading in scale-free networks. Phys. Rev. Lett. 86, 3200-3203.

[28] Serrano, M. and Boguñá, M. (2006). Percolation and epidemic thresholds in clustered networks. Phys. Rev. Lett. 97, 088701.

[29] Trapman, P. (2007). On analytical approaches to epidemics on networks. Theoret. Pop. Biol. 71, 160-173.

[30] Van den Berg, J., Grimmett, G. R. and Schinazi, R. B. (1998). Dependent random graphs and spatial epidemics. Ann. Appl. Prob. 8, 317-336. 